\title{
Statics Analyses of a Riveting Robot based on 6-SPU Parallel Mechanism
}

\author{
Zheng LIANG $^{1}$, Yong $\mathrm{XU}^{2}$ and Yeping LV \\ ${ }^{2}$ Yong $\mathrm{XU}^{2}$,College of Mechanical and Automotive Engineering, Shanghai University of Engineering Science, shanghai , 201620,
}

\begin{abstract}
A new walking riveting robot based on 6-SPU parallel mechanism is designed to meet the needs of high precision and high efficiency machining for large, thin-walled and complex curved surface workpieces in aviation industry and automotive industry. After modeling and solving the position inverse solution of the robot, the riveting trajectory planning is carried out, and the correct positions and orientations of riveting holes are determined. For the workpiece with large thin-walled cylindrical surface, the riveting hole process of the robot is simulated by finite element analysis software, the maximum deformations in all orientations and the maximum equivalent stresses of the moving platform corresponding to riveting points are obtained. The above results provide the necessary theoretical basis for the structure optimization and performance analyses of the automatic riveting robot.
\end{abstract}

\section{Introduction}

Stiffness is an important performance indicator for the robot's work unit. The stiffness of the robot varies from its configuration ${ }^{[1]}$, so it is necessary to quickly predict whether the stiffness of the mechanism meets the operational requirements during the design phase of the mechanism, in order to provide the necessary theoretical basis for the detailed design of the mechanical structure. In this paper, a new type of walking riveting robot based on 6-SPU parallel mechanism is taken as the research object, and it is analyzed by multi-software joint method: the trajectory planning of robot riveting operation is carried out based on position inverse solution, and the position of each riveting point of the mechanism is obtained. The robot is three-dimensionally modeled by SolidWorks, and then static analysis is performed using the finite element analysis software ANSYS.

\section{Robot configuration}

In order to complete the riveting and hole-making task of the large-scale thin-walled free-form surface, this paper designs a walking type based on 6-SPU (S, P and $U$ denotes spherical pair, active prismatic pair and universal joint respectly) six-degree-of-freedom parallel mechanism.

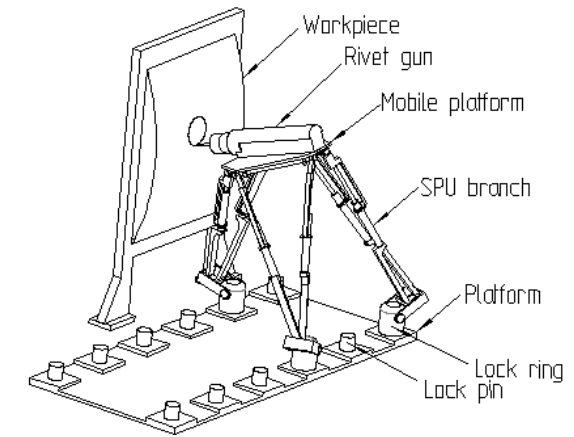

Figure 1. Schematic diagram of riveting robot processing

The Riveting robot ${ }^{[2]}$ presented by our research group is shown in Fig.1. The schematic diagram of the mechanism is shown in Fig.2. Each branch (walking leg) is a 2-SPU two-degree-of-freedom parallel mechanism with a composite spherical pair $\mathrm{S}$.

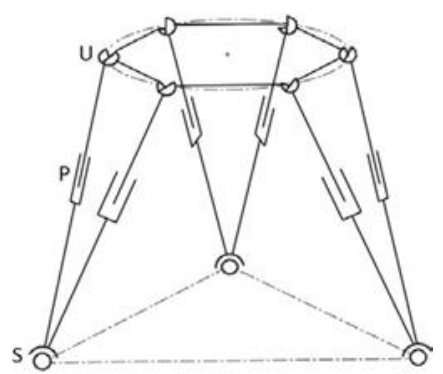

Figure 2. Schematic diagram of 6-SPU mechanism

\section{Riveting trajectory planning based on the inverse position solution}

\subsection{Inverse position solution}




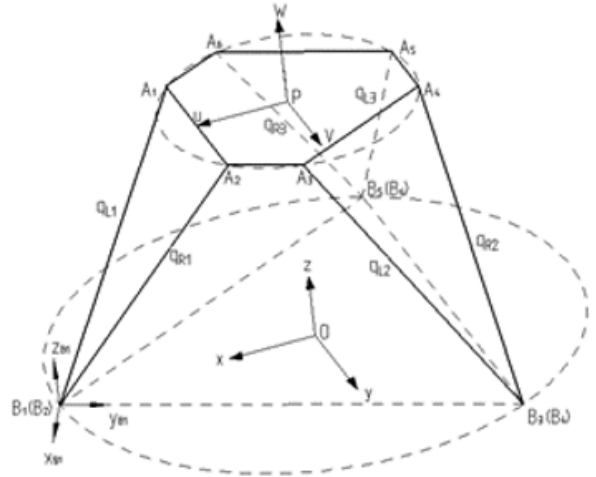

(a) Mechanism coordinate system setting

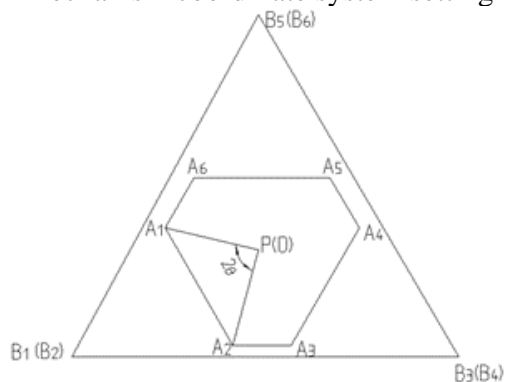

(b) Initial layout of robot platforms

Figure 3. Mechanism coordinate system setting As shown in Fig. 3., the fixed coordinate system $\{\mathrm{O}-\mathrm{xyz}\}$ is established: the coordinate origin $\mathrm{O}$ is the centroid of the fixed platform, the $\mathrm{x}$-axis is from $\mathrm{O}$ to the composite spherical sub-center $\mathrm{B}_{1}\left(\mathrm{~B}_{2}\right)$, the $\mathrm{z}$-axis is perpendicular to the platform upward, and the y-axis Determined by the right- hand rule. Establish the dynamic coordinate system $\{\mathrm{P}-\mathrm{uvw}\}$ : the origin of the coordinate $\mathrm{P}$ is the centroid of the moving platform, and the $\mathrm{u}$-axis is pointed by $\mathrm{P}$ to the midpoint of the connection between the two U-shaped centroids $A_{1}$ and $A_{2}$ on the moving platform, and the $\mathrm{W}$-axis is vertically moved up, the v-axis is determined by the right-hand rule. The initial configuration of the mechanism is parallel to the motion and the fixed platform, and the coordinate axes of the dynamic and fixed coordinate systems are respectively parallel.

From the above, the position vector $\boldsymbol{A}_{\boldsymbol{i}}^{\boldsymbol{P}}$ of each U-shaped centroid $A_{i \text { in the moving coordinate system }}$ $\{\mathrm{P}-\mathrm{uvw}\}$ on the moving platform and the position vector $B_{j}^{o}$ of each composite spherical sub-center ${ }^{B_{j}}$ in the fixed coordinates system $\{\mathrm{O}-\mathrm{xyz}\}$ in the fixed platform is known.

The 6-S $\underline{P U}$ mechanism satisfies the following position vector equation

$$
B_{i}^{O}+q_{i}=P^{O}+P A_{i}^{O}
$$

In the middle, $\boldsymbol{P} \boldsymbol{A}_{\boldsymbol{i}}^{\boldsymbol{O}}=\boldsymbol{R} \cdot \boldsymbol{P} \boldsymbol{A}_{\boldsymbol{i}}^{\boldsymbol{P}}, \boldsymbol{P} \boldsymbol{A}_{\boldsymbol{i}}^{\boldsymbol{P}}, \boldsymbol{P} \boldsymbol{A}_{\boldsymbol{i}}^{\boldsymbol{O}}$ is the vectors of $P A_{i}$ in the moving and fixed platform coordinate system, respectively. Position vector of moving platform centroid in fixed coordinate system $P^{O}=(x, y, z) ; \quad \boldsymbol{q}_{\boldsymbol{i}}$ is a single drive displacement vector in the branch; ${ }_{\boldsymbol{O}}^{\boldsymbol{P}} \boldsymbol{R}_{\boldsymbol{x y z}}(\boldsymbol{\alpha}, \boldsymbol{\beta}, \boldsymbol{\gamma})$ is the attitude transformation matrix of the moving coordinate system relative to the fixed coordinate system

$$
\begin{aligned}
& { }_{o}^{P} R_{x y z}(\alpha, \beta, \gamma) \\
& =\left(\begin{array}{ccc}
c \beta c \gamma & s \alpha s \beta c \gamma-c \alpha s \gamma & c \alpha s \beta c \gamma+s \alpha s \gamma \\
c \beta s \gamma & s \alpha s \beta s \gamma+c \alpha c \gamma & c \alpha s \beta s \gamma-s \alpha c \gamma \\
-s \beta & s \alpha c \beta & c \alpha s \beta
\end{array}\right)
\end{aligned}
$$

Here, $s \alpha$ and $\operatorname{co}$ represent $\sin \alpha$ and $\cos \alpha$, and the rest are similar. A single drive displacement vector $\boldsymbol{q}_{\boldsymbol{i}_{\text {can be }}}$ obtained from equation (1).

$$
q_{i}=P^{O}+P A_{i}^{O}-B_{i}^{O}
$$

\subsection{Riveting trajectory planning}

In this paper, the 6-SPU robot performs the riveting and hole-making task on the surface of a large-scale thin-walled cylindrical surface workpiece. The space curve formed by the multiple riveting holes is the end track of the riveting gun.

Based on Frenet-Serret vector space [3] in the differential geometry curve theory, a riveting point coordinates system can be established. The Matlab software is used to calculate the unit tangent vector $\overrightarrow{\boldsymbol{e}}_{\boldsymbol{\tau}}$ at the points of the trajectory, the unit normal vector $\overrightarrow{\boldsymbol{e}}_{\boldsymbol{n}}$ and the unit secondary normal vector $\overrightarrow{\boldsymbol{e}}_{\boldsymbol{b}}$. In order to ensure the machining accuracy of the riveting hole, the advancing direction of the rivet gun must be consistent with the $\overrightarrow{\boldsymbol{e}}_{\boldsymbol{\tau}}$ direction of the riveting point coordinates system of the workpiece surface, and the rivet gun main axis direction must be consistent with the $\overrightarrow{\boldsymbol{e}}_{\boldsymbol{b}}$ direction of the riveting point coordinate system. The coordinates axis direction of each riveting point is shown in Fig. 4. The position coordinates of each riveting point and the corresponding attitude angle of the main axis of the riveting gun is shown in Table 1.

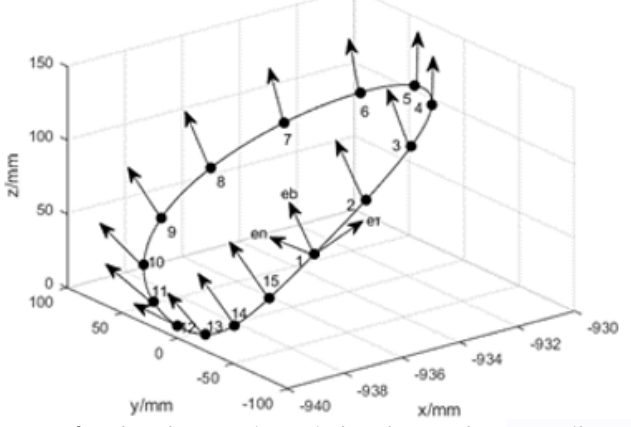

Figure 4. Riveting path and riveting point coordinates

Table 1. Riveting hole positions and riveting gun attitude angles

\begin{tabular}{|l|l|c|}
\hline No. & $\begin{array}{l}\text { Riveting hole } \\
\text { positions } / \mathrm{mm}\end{array}$ & $\begin{array}{c}\text { Riveting gun } \\
\text { attitude angles/ }\end{array}$ \\
\hline
\end{tabular}




\begin{tabular}{|c|c|c|}
\hline 1 & $(-935.5,-54.8,89.0)$ & $(175.1,87.3,85.9)$ \\
\hline 2 & $(-933.4,-40.2,109.2)$ & $(174.6,89.0,84.6)$ \\
\hline 3 & $(-931.9,-18.7,121.7)$ & $(173.1,89.9,83.1)$ \\
\hline 4 & $(-931.5,6.2,124.3)$ & $(172.6,89.9,82.6)$ \\
\hline 5 & $(-932.5,29.4,116.6)$ & $(173.7,90.3,83.7)$ \\
\hline 6 & $(-934.4,48.4,100.0)$ & $(175.0,91.8,85.4)$ \\
\hline 7 & $(-936.5,58.6,77.2)$ & $(174.8,93.2,86.0)$ \\
\hline 8 & $(-938.3,58.7,52.3)$ & $(174.8,93.3,86.0)$ \\
\hline 9 & $(-939.3,48.6,29.5)$ & $(176.2,91.7,86.7)$ \\
\hline 10 & $(-939.6,30.1,12.7)$ & $(178.2,90.4,88.2)$ \\
\hline 11 & $(-939.7,6.3,4.9)$ & $(179.5,89.9,89.5)$ \\
\hline 12 & $(-939.7,-18.4,7.5)$ & $(178.9,89.9,88.9)$ \\
\hline 13 & $(-939.5,-40.1,20.0)$ & $(177.3,89.0,87.5)$ \\
\hline 14 & $(-938.9,-54.8,40.2)$ & $(175.3,87.2,86.2)$ \\
\hline 15 & $(-937.5,-60.0,64.6)$ & $(174.7,86.5,86.0)$ \\
\hline
\end{tabular}

\section{Finite element analyses}

\subsection{Finite element preprocessing}

The stiffness model of the parallel mechanism is a function of the configuration. When using the finite element software to analyze the parallel mechanism, the finite element analysis of the parallel mechanism should be performed for different configurations ${ }^{[4]}$. The solid model of the organization is established by Solidworks, and the parallel mechanisms are assembled according to the actual situation. From Table 1, the positional parameters of the parallel mechanism are calculated by position inverse solution, the parallel mechanism is adjusted to the required pose and imported into the Ansys Workbench, and the connection conditions are set according to the motion pair of the parallel mechanism. The material of the parallel mechanism is set to structural steel, the elastic modulus $\mathrm{E}=200 \mathrm{GPa}$, the density is 7850 kg.m-3, the Poisson's ratio $\gamma=0.3$, and the yield limit $\delta=350 \mathrm{MPa}$. The whole machine model uses a tetrahedral unit to divide the grid. The quantity of the elements is 27370 , and the quantity of nodes is 80092 , as shown in Fig.5. The rivet gun is subjected to an axial working load $\mathrm{F}=110 \mathrm{~N}$ along the axis of the rivet gun during operation.

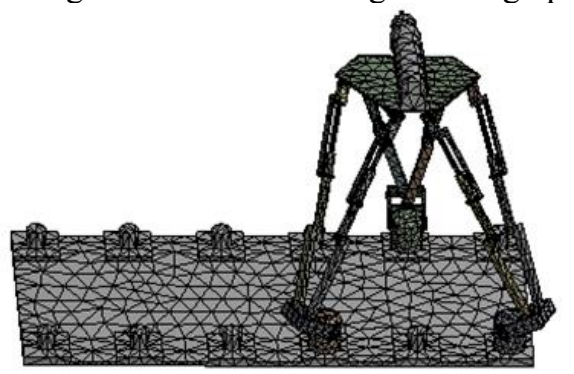

Figure 5. Robot meshing model

\subsection{Statics analysis}

Statics analysis can comprehensively and intuitively evaluate the deformation and stress distribution of the mechanism ${ }^{[5-7]}$. Static analysis was performed on the 6-SPU mechanism, and the results are shown in Fig. 6-7. During the analysis process, the deformation and equivalent stress generated by the mechanism in the working state are mainly distributed on the moving platform and the driving rod, while on the other parts are small.

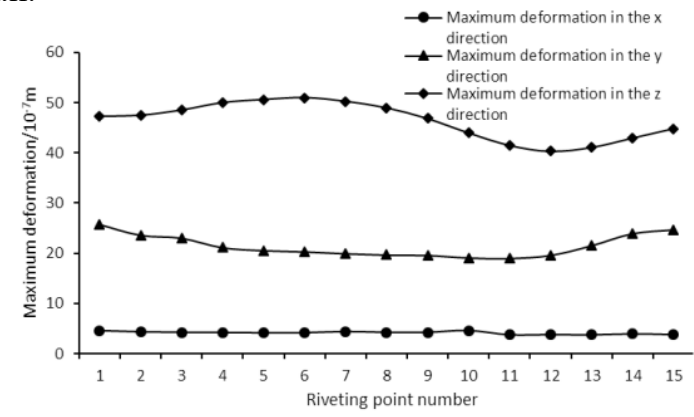

Figure 6. Maximum deformation of the moving platform in the $\mathrm{x}, \mathrm{y}$, and $\mathrm{z}$ directions

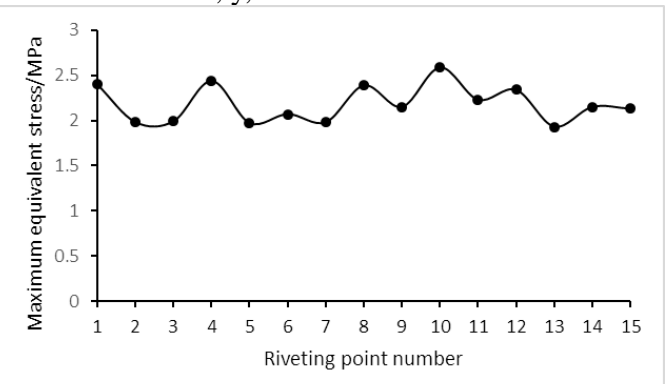

Figure 7. Robot equivalent stresses

Fig.6. shows that the maximum deformation of the mechanism in the $\mathrm{z}$ direction is higher than the $\mathrm{x}$ and $\mathrm{y}$ directions, indicating that the stiffness of the mechanism in the $\mathrm{z}$ direction is lower than the other two directions, that is, the bearing capacity in the $\mathrm{z}$ direction is poor; the minimum amount of deformation of the mechanism in the $\mathrm{x}$ direction indicates that the stiffness of the mechanism in the direction is better, that is, the bearing capacity in the $\mathrm{x}$ direction is stronger than other directions. It can be seen from Fig. 7. that the maximum equivalent stress of the mechanism is less than the yield limit of the material, which satisfies the requirements.

\section{Conclusions}

For the high-end manufacturing industry, this paper proposes and deeply studies a new walking riveting robot based on 6-SPU parallel mechanism. The specific results and conclusions are as follows:

(1) Modeling and solving the inverse solution of the mechanism position, based on which the robot riveting trajectory planning is completed, and the precise trajectory of the end of the rivet gun and the correct riveting operation posture are determined.

(2) For the large-scale thin-walled cylindrical workpiece, the finite element analysis software is used to simulate the robot riveting operation, and the maximum deformation and equivalent stress in the $\mathrm{x}, \mathrm{y}$, and $\mathrm{z}$ directions of the moving platform corresponding to each riveting point station were obtained.

The above results prove the rationality of the mechanism configuration, position inverse solution and trajectory planning model proposed in this paper. The finite element analysis results also show that the mechanism meets the work intensity and accuracy requirements. The related results provide the necessary 
theoretical basis for structural optimization and performance analysis of automatic riveting robots.

\section{References}

1. Wang Manxin, et al. Journal of Mechanical Engineering47, 9(2011).

2. Liu Wencai, Xu Yong. Mechanical Design and Research34, 76(2018).

3. Ying Can. Research on trajectory optimization of cooperative motion of welding robot workstation [D]. South China University of Technology, 2013.

4. Wang Youyu. Journal of Mechanical Engineering08, 13(2008).

5. Zhang Jun, He Fang. Equipment Manufacturing Technology10, 15(2016).

6. Cao Luhai, et al. Machine Tool\&Hydraulics43, 127(2015)

7. Li Zhihua, Yu Jun.Transactions of the Chinese Society of Agricultural Machinery46, 364(2015). 